\title{
The Recoil Polarization Experiments at Jefferson Lab
}

\section{F. Perdrisat*}

College of William and Mary, Williamsburg, VA 23187, USA

E-mail:perdrisa@jlab.org

\section{Punjabi}

Norfolk State University, Norfolk VA 23804, USA

E-mail:punjabi@jlab.org

\section{M.K. Jones}

Thomas Jefferson National Accelerator Facility, Newpoprt News, VA 23606, USA

E-mail:jones@jlab.org

\begin{abstract}
The use of polarization observables to obtain the form factors of the nucleon has resulted in a drastic change in the way we understand the structure of both proton and neutron. In particular, the measurement of the two non-zero components of the recoil proton polarization in the elastic $\vec{e} p \rightarrow$ $e^{\prime} \vec{p}$ reaction in three experiments at JLab, have resulted in determining the ratio $G_{E p} / G_{M p}$ up to a $\mathrm{Q}^{2}$ of $8.5 \mathrm{GeV}^{2}$. These experiments were done with two different proton polarimeters, capable of good performance from a few $\mathrm{GeV} / \mathrm{c}$ to $5.4 \mathrm{GeV} / \mathrm{c}$ proton momentum Ref. [1, 2, 3, 4, 5, 6]. A planned experiment, which will become possible with $11 \mathrm{GeV}$ electrons in Hall A after the JLab energy upgrade, is being designed and should reach proton momenta of $8 \mathrm{GeV} / \mathrm{c}$, corresponding to $\mathrm{Q}^{2}=15 \mathrm{GeV}^{2}$, Ref. [7]. It will require a new polarimeter, to be added to the Super Bigbite Spectrometer facility (SBS). The evolution of the design and performance of the two polarimeters will be discussed in some details.
\end{abstract}

PSTP2013: The 2013 International Workshop on polarized sources, targets and polarimetry. University of Virginia, Charlottesville, Virginia, September 9-13, 2013.

\footnotetext{
*Speaker.
} 
The use of the recoil polarization technique to obtain the elastic form factors of proton and neutron at large $\mathrm{Q}^{2}$ has become possible with the development of highly polarized and high luminosity electron beams in the multi-GeV energy range; in particular at the Jefferson National Accelerator Facility (TJNAF or JLab) electron accelerator (or CEBAF). It is one of the two double-polarization techniques that can be used to this end, the other being polarized electron on a polarized target (proton or neutron), measuring the asymmetry. Here we will discuss the evolution of the recoil polarization technology, which started in 1998, with the first experiment to obtain the elastic electric, $G_{E p}$, to magnetic, $G_{M p}$, Sachs form factor ratio $G_{E p} / G_{M p}$ by the recoil polarization method at JLab Halls A and C.

The first suggestion that double polarization would be a better way to obtain nucleon form factors in elastic ep scattering goes back to a paper by Akhiezer and Rekalo [8]. Until the late 1990 's the only way to obtain the proton form factors was through measurement of the differential cross section. In Born approximation, that is, assuming that single virtual photon exchange is the dominant process in elastic ep scattering, the ep cross section is the product of the Mott cross section times a factor $\varepsilon G_{E p}^{2}\left(Q^{2}\right)+\tau G_{M_{p}}^{2}\left(Q^{2}\right) . Q^{2}$ is the negative of the square of the invariant energy transfer, $Q^{2}=-\left(\omega^{2}-q^{2}\right)$, with $(\omega, \vec{q})$ the virtual photon four-momentum. $\varepsilon=(1+2(1+$ $\left.\tau) \tan ^{2} \frac{\theta_{e}}{2}\right)^{-1}$ and $\tau=Q^{2} / 4 M_{p}^{2}$ are the kinematic factor (or polarization of the virtual photon) and the dimension-less momentum transfer squared, respectively. The well known Rosenbluth separation method consists then in measuring the differential cross section for several values of $\varepsilon$ by changing both beam energy and detector angle, while maintaining $Q^{2}$ constant.

In Born approximation and for polarized incident electrons, the recoiling proton is polarized, with a longitudinal component along the final proton momentum, $\mathrm{P}_{\ell}$, and a transverse component in the reaction plane, $\mathrm{P}_{t}$. Measuring these two components determines $G_{E p}$ and $G_{M p}$, provided one knows accurately enough the beam polarization and the analyzing power of the polarimeter used for the measurement of the proton polarization.

Here we discuss the technique related to recoil polarization transfer as used for elastic electron scattering on a free proton, as was developed in the course of about 10 years to obtain the form factor ratio over the the large $Q^{2}$ range 0.5 to $8.5 \mathrm{GeV}^{2}$, and the future $12 \mathrm{GeV}^{2}$, after completion of the Jefferson Lab $12 \mathrm{GeV}$ upgrade.

In the energy range of interest here, i.e. proton momenta from about $0.7 \mathrm{GeV} / \mathrm{c}$ to $7-8 \mathrm{GeV} / \mathrm{c}$, the proton polarization components perpendicular to the proton momentum can be obtained by scattering the proton in an analyzer block made preferentially of $\mathrm{CH}_{2}$, and observing the azimuthal asymmetry with appropriate tracking detectors downstream of the analyzer. The asymmetry in such collisions is caused by the $\vec{L} \cdot \vec{S}$ coupling in NN interaction. Following the Basel convention (1960), spin- particles with spin up scatter preferentially to the left if the analyzing power, $A_{y}$, is positive. As only components perpendicular to the proton momentum can be determined with this method, it is necessary to rotate the longitudinal polarization component so as to have as large as possible a component normal to both the proton momentum and the transverse polarization component. This rotation can be obtained with a dipole, which, at least in first order, does not precess the transverse component, if the B-field is perpendicular to the proton momentum, as illustrated in Fig. 1. The azimuthal distribution of the scattered proton will then be $I(\phi)=I_{0}\left(1+A_{y}(\theta) \vec{P}^{f p p} \cdot \vec{n}\right)$, where $\theta$ and $\phi$ are the polar and azimuthal scattering angles, respectively; $\vec{n}$ is the normal to the ep-scattering plane and $\vec{P}^{f p p}$ is the proton polarization vector. Fig. 2 is a schematic illustration of a polarimeter, 


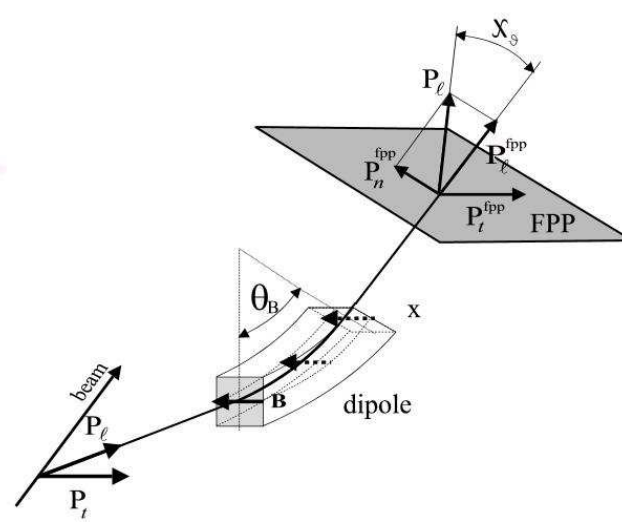

Figure 1: Precession of $P_{\ell}$ in typical recoil polarization experiment.

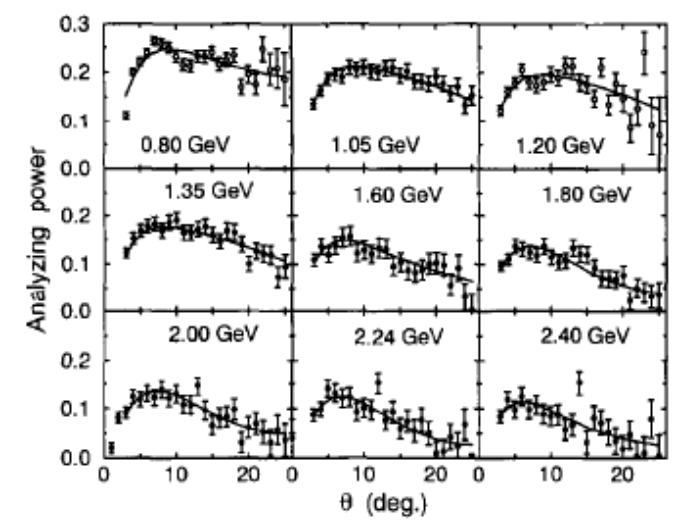

Figure 3: Analyzing powers $\mathrm{A}_{y}$ for $\mathrm{pC}$ obtained in 1995 in Saclay; the fits are 8-parameter polynomials. The maximum energy would correspond to a $\mathrm{Q}^{2}$ of $4.5 \mathrm{GeV}^{2}$ in elastic ep.

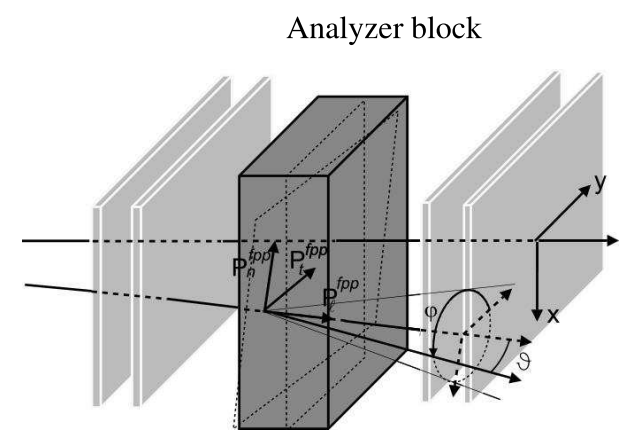

Front tracker

Back tracker

Figure 2: Determination of the azimuthal asymmetry in a polarimeter

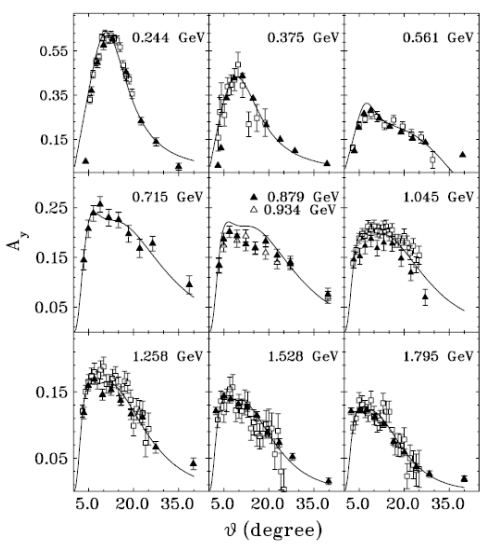

Figure 4: pC Analyzing powers obtained from the first GEp experiment in 1998; some of the data points from Fig. 3 are included.

with its front- and back tracking detectors determining $\theta$ and $\phi$. The expected azimuthal distribution will then be of the form $\left.N^{+}(\theta, \phi)=N_{0} \frac{\varepsilon(\theta, \phi)}{2 \pi}\left[1+A_{y} P_{t}^{f p p} \cos \phi-A_{y} P_{n}^{f p p} \sin \phi\right)\right]$ for positive helicity of the electron beam, where $P_{t}^{f p p} \sim P_{t}^{\text {target }}$ and $P_{n}^{f p p} \sim P_{\ell}^{\text {target }} \sin \chi$ are the normal and transverse polarization components at the FPP, $\chi=\gamma \Theta_{\text {bend }}\left(\mu_{p}-1\right)$ is the spin precession angle for the dipole bend angle $\Theta_{b e n d}$, and $\varepsilon(\theta, \phi)$ is the efficiency of the polarimeter; its $\phi$-dependence is the results of instrumental asymmetries, which can be mostly canceled by forming the helicity difference $N^{+}-N^{-}$.

The first polarization transfer experiment at JLab was inspired by the pioneering theoretical 


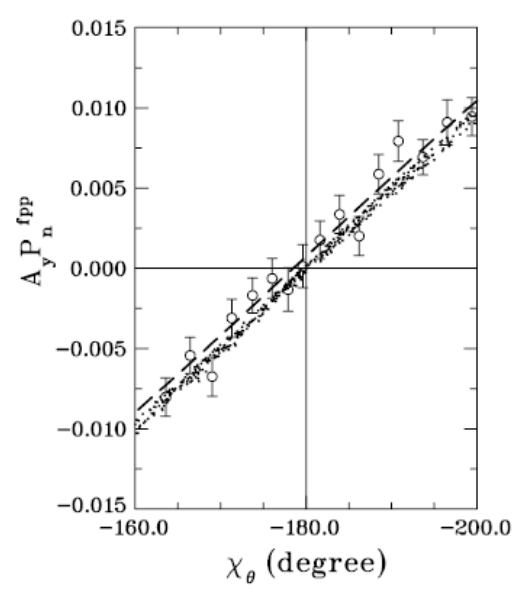

Figure 5: Zero crossing of precessed longitudinal component of the polarization; the small dots are simulation data. The dashed line is a fit to the data, and the solid line fit to the simulation.

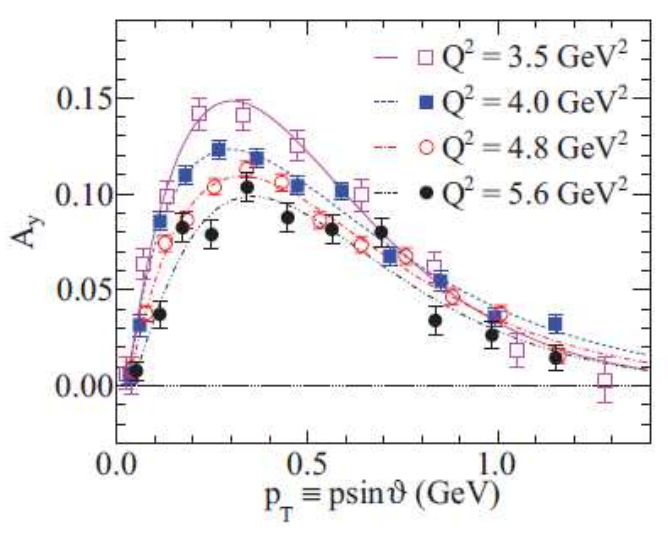

Figure 6: Analyzing powers from GEp(2) using $\mathrm{CH}_{2}$ as analyzer material. The maximum $\mathrm{Q}^{2}$ corresponds to $3.8 \mathrm{GeV} / \mathrm{c}$ momentum.

work of several authors $[8,9,10]$. It was based on analyzing power data obtained at the accelerator SATURNE with the polarimeter POMME (Polarimeter Mobile a Moyenne Energy), obtained with polarized proton beams of energy up to $2.4 \mathrm{GeV}(3.2 \mathrm{GeV} / \mathrm{c})$ ([11]) prior to the $\mathrm{GEp}(1)$ experiment at Jefferson Lab (see Fig. 3). The polar angle distributions of $A_{y}$ obtained in the GEp(1) experiment are plotted in Fig. 4, from Ref. [2]. In this experiment the ratio $G_{E p} / G_{M p}$ was measured directly, for the first time, from the ratio $P_{t} / P_{\ell}$, as had been proposed by Punjabi and Perdrisat in the original GEp(1)-proposal [12]. The main advantage of this method is that the ratio is independent of the beam polarization as well as the polarimeter $A_{y}$. And of course, as two physics quantities have been measured, two results can be obtained: the second is the analyzing power. $A_{y}$ determines the error bars, as in first approximation $\Delta P_{t}^{f p p}=\Delta P_{\ell}^{f p p}=\sqrt{\left(\frac{2}{\varepsilon A_{y}^{2} N}\right)}$, where $\mathrm{N}$ is the number of ep events, $A_{y}$ an average analyzing power, and $\varepsilon$ the efficiency of the polarimeter.

Until the first GEp experiment at JLab, the proton form factors had been obtained almost exclusively by Rosenbluth separation. These measurement showed that, individually, the $Q^{2}$ dependence of the two form factors was similar to the dipole form factor $G_{D}=\left(1+\frac{Q^{2}}{0,71}\right)^{-2}$, resulting in an approximately constant $G_{E p} / G_{M p}$ ratio. The results of GEp(1) strongly suggested (see Ref. [2]) that the recoil polarization results may not be in agreement with the Rosenbluth data base. One possible source of systematic error could have been in the method used to reconstruct the target polarization components, $\left(P_{t}^{t g t}, P_{\ell}^{t g t}\right)$ from the measured focal plane polarimeter polarization components $\left(P_{t}^{f p p}\right.$, $\left.P_{n}^{f p p}\right)$. The data in Fig. 5 were obtained by choosing a kinematics such that the precession in the HRS dipole would be near $180^{\circ}$. Events in the acceptance provide a range of precession angles and the values of the asymmetry amplitude $A_{y} P_{n}^{f p p}$ do indeed go through zero very close to $180^{\circ}$ precession angle, corresponding to $P_{n}^{f p p}=0$, validating the calculation of the spin precession.

The results of the second GEp experiment at JLab are published in Ref. [3]. The data have been re-analyzed since and re-published in Ref. [4]. The analyzing power results of GEp(2) are shown in Fig. 6; note that the maximum of $\mathrm{A}_{y}$ appears at a nearly constant transverse momentum 
$\mathrm{p}_{T}$ of $\approx 0.3-04 \mathrm{GeV} / \mathrm{c}$ over the range of proton momentum 2.6 to $3.8 \mathrm{GeV} / \mathrm{c}$. These results confirm the data obtained in a calibration experiment at JINR/Dubna, (see Ref. [13]), which was necessary to get the GEp(3) experiment approved by the JLab PAC. An apparent "scaling" of $A_{y}^{\max }$ versus 1/p, seen in Fig. 7, was observed for the first time in Ref. [13].

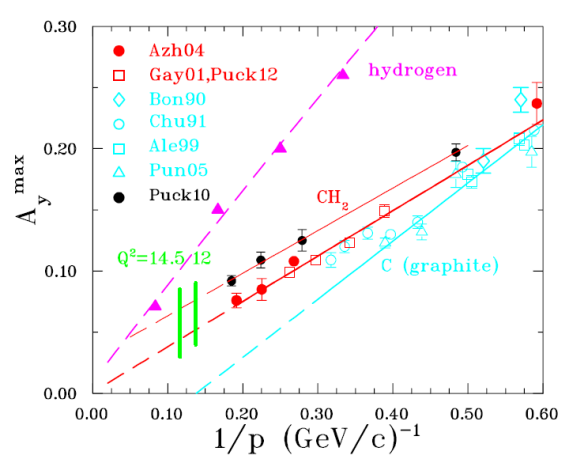

Figure 7: The data of Ref. [13] indicated the scaling relation for the maximum value of $A_{y}$ versus $1 / p$. The scatter of the data from different experiments is a reflexion of the efficient of single-track identification.

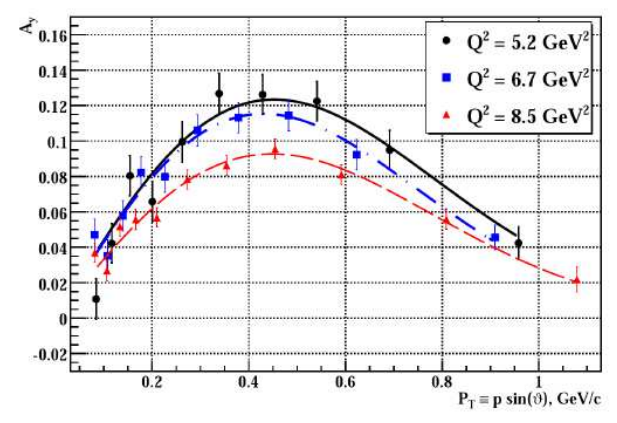

Figure 8: Analyzing powers for $\mathrm{CH}_{2}$ from GEp(3), using $\mathrm{CH}_{2}$ as analyzer material; the highest $\mathrm{Q}^{2}$ value corresponds to a proton momentum of $5.4 \mathrm{GeV} / \mathrm{c}$ [14].

So far the tracking of the polarimeters used at JLab did not allow for particle identification. As the proton energy increases, the fraction of single-track events decreases, approximately like the ratio of the elastic to inelastic total cross section. The undesirable effect of the inelastic contribution, which can only partially be suppressed by selecting single-track events (some inelastic channels results in a single charged final state, example $p n \rightarrow p n \pi^{0}$ ), is a decrease of the effective analyzing power $A_{y}$. In future experiments, better tracking resolution and crude measurement of energy behind the polarimeters should increase the effective analyzing power: $\mathrm{HCal}$ in the SBS setup under construction for $\operatorname{GEp}(5)$ will allow a crude energy determination.

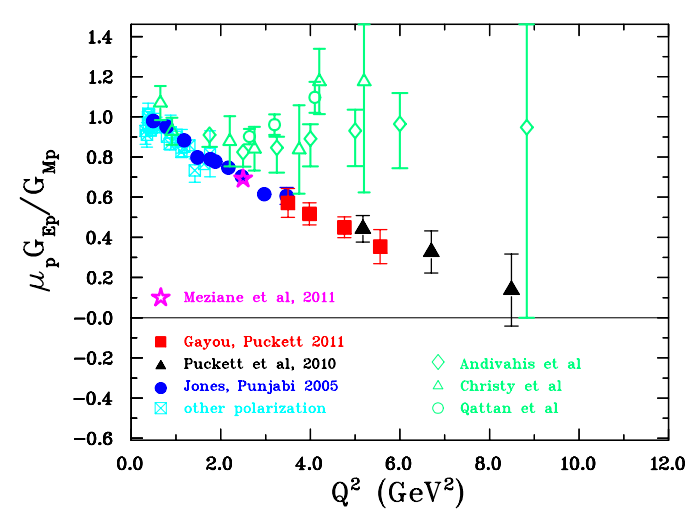

Figure 9: Results of the three JLab GEp experiments obtained by the recoil polarization method.

The third GEp experiment at JLab in 2007-8 used a double polarimeter installed in the Hall C 
HMS spectrometer; the polar angle dependence of the analyzing powers obtained, over the proton momentum range 3.6 to $5.4 \mathrm{GeV} / \mathrm{c}$ are shown in Fig. 8.

Fig. 9 shows all values of $\mu_{p} G_{E p} / G_{M p}$ obtained in recoil polarization experiments at JLab so far; as well as values obtained in a number of other experiments to check the polarimeter performance. The results of the 3 most recent Rosenbluth experiments $([15,16,17]$ are also shown for comparison .

\section{Conclusions}

Recoil polarization experiments became possible with JLab; the technique had been first tested at Bates at $\mathrm{Q}^{2}<0.5 \mathrm{GeV}^{2}$ in 1996 [18].

Results of recoil polarization experiments were unexpected, showing an irreducible difference between double polarization results and Rosenbluth separation results.

It is now commonly assumed that the difference is due primarily to incomplete radiative corrections to the cross section data, including double virtual photon exchange. The size of the twophoton exchange has yet to be determined experimentally; the e+/e- cross section ratio recently measured in three Laboratories should resolve the puzzle (although this ratio is quite sensitive to radiative corrections too!).

The authors acknowledge the support of NSF grant 1066374 (CFP), and DOE grant DE-FG0289ER40525 (VP).

\section{References}

[1] M.K. Jones et al. P.R.L. 84, 1398 (2000).

[2] V. Punjabi et al. P.R. C 71055202 (2005), errat. ibid C71,069902 (2005).

[3] O. Gayou et al., P.R.L. 88, 092301 (2002).

[4] A.J.R. Puckett et al., P.R. C 85 (2012), 045203.

[5] A.J.B. Puckett et al., P.R.L. 104, 242301 (2010).

[6] M.Meziane et al. P.R.L. 106 (2011) 132501.

[7] C.F. Perdrisat et al., proposal PR12-07-109, JLAB PAC 32 (2007)

[8] A.I. Akhiezer and M.P. Rekalo, Sov. J. Part. Nucl. 3, 277 (1974).

[9] N. Dombey, Rev. Mod. Phys, 41, 236 (1969).

[10] R.G. Arnold, C.E. Carlson and F. Gross, P.R. C 23, 363 (1981).

[11] E. Chung et al. N.I.M. in P.R. A363 (1995) 561.

[12] C.F. Perdrisat and V. Punjabi, "Electric Form Factor of the Proton by Recoil Polarization, JLab PAC6, (1989).

[13] L.S. Azhgirey et al, N.I.M. in P.R. A 538 (2005), 431.

[14] A.J.R. Puckett, unpublished PhD thesis, MIT (2009).

[15] L. Andivahis et al., P. R. D 50, 5491 (1994).

[16] Christy et al., P.R. C 70, 015206 (2004).

[17] I.A. Qattan I. A. et al., P. R. L. 94, 142301 (2005).

[18] B.D. Milbrath et al. P.R.L. 80 (1998), 452; erratum P.R.L. 82 (1999),221. 